Yann HAMEL, «Le sport qu'on lit », @nalyses, automne 2006

\title{
Yann HAMEL
}

\section{Le sport qu'on lit}

Le dimanche 23 avril 1916, Barcelone fut le théâtre d'un combat de boxe opposant Jack Johnson, $110 \mathrm{~kg}$, ex-champion du monde, et Fabian Lloyd, alias Arthur Cravan, poète. Révélant dans sa flagrante évidence le déséquilibre entre champions du muscle et des lettres, la rencontre, qui se termina au sixième round « dès que Johnson décida d'en finir, d'une belle droite » (p. 9), incite Jean Durry à (se) poser, en entrée de jeu d'un collectif portant sur la mise en texte des compétitions sportives, une question pour le moins inusitée : «Lier à toutes forces sport et littérature, n'est-ce pas une imposture? »

S'il se trouvait, encore aujourd'hui, des chercheurs en sciences humaines ou des lettrés pour douter qu'une telle question doive recevoir une réponse négative, la "conférence en guise d'ouverture » (p. 9-35) de Durry suffirait à prouver, avec éclat, que la mise en texte de ce qui se passe dans les stades ou sur les pistes est bel et bien une réalité intéressant à la fois les études littéraires, la sociologie, les sciences politiques, ainsi que différentes branches de l'histoire, celle du sport proprement dit bien sûr, mais aussi, pour ne nommer que quelques exemples, les histoires du vêtement, des mœurs, des idées et de la culture. D'Ompdrailles-le-tombeau-des-lutteurs (1867), premier roman sportif français dû à Léon Cladel, jusqu'aux incontournables travaux de Pierre Charreton ${ }^{1}$, en passant par un catalogue encyclopédique d'anthologies, d'essais en tous genres et de fictions prenant pour thème la boxe, le cyclisme, le football et l'athlétisme, Durry couvre, à lui seul, une part considérable de ce vaste terrain où la plume se collette avec l'exploit physique.

\footnotetext{
${ }^{1}$ Pierre Charreton, Les fêtes du corps. Histoire et tendances de la littérature à thème sportif en France: 1870-1970, Saint-Étienne, Université de Saint-Étienne, 1985, et Le sport, l'ascèse, le plaisir. Éthique et poétique du sport dans la littérature française moderne, SaintÉtienne, Centre interdisciplinaire d'étude et de recherche sur l'expression contemporaine/Université Jean-Monnet, 1990.
} 
Les dix-sept autres chercheurs rassemblés dans Écrire le sport ne sont pas en reste sur le plan de l'érudition. Le lecteur qui entreprendra la traversée de ce volume rencontrera, sur son chemin, des gens d'aussi bonne compagnie que Guy de Maupassant, Tristan Bernard, Pierre Loti, Jack London, Louis Hémon, Paul Morand, Marcel Aymé, Arthur Conan Doyle, Jean Giraudoux, Ernest Hemingway, Dino Buzzati et Norman Mailer. Il fera, ici et là, un bout de chemin en compagnie de l'inéluctable Coubertin, d'Henri de Montherlant, chantre des jeunes corps athlétiques, et d'Antoine Blondin, chroniqueur «buissonnier» du Tour de France. Il tombera sur les mémoires d'anciens athlètes et de responsables de fédérations sportives en mal de disculpation, sur les récits autobiographiques de plusieurs centaines d'alpinistes, sur les premiers balbutiements du jiu-jitsu français, sur les essais sociologiques de Pierre Bourdieu et de Bernard Jeu ${ }^{2}$, sur une douzaine de polars des années 1990 qui dépeignent les richissimes et malfamés milieux du sport professionnel, sur les mythologies de Barthes, sans oublier moult pages de L'Auto, de L'Équipe et de la puissante presse sportive italienne. Il passera et repassera à maintes reprises par les mémorables jeux de la VIII olympiade de Paris (1924), où les performances du corps athlétique donnèrent lieu à une effervescence poétique et intellectuelle sans précédent. Pour peu qu'il ne soit pas déjà un vieux spécialiste de la crapahute en sentiers sportivo-lettrés, il fera en outre connaissance avec Roger Vailland, Joseph Jolinon et, surtout, Théodore Chèze, auteur de Claude Lenoir (1907), ce « chef d'œuvre inconnu » du roman cycliste (p. 17).

À cette diversité jubilatoire des disciplines sportives, des écrivains, des époques, des pays et des genres du discours convoqués se superpose une diversité non moins grande des angles d'approches privilégiés et des types d'analyses pratiquées par les auteurs des différents articles. Les deux grandes catégories entre lesquelles les textes ont été répartis, «Écritures du sport» (p. 37-115) et «Sport et littérature » (117-244), font se côtoyer enquêtes lexicographiques savantes, questionnements

\footnotetext{
2 Il faut préciser que Norbert Elias et Eric Dunning ne sont pas de la partie, ce qui n'est pas sans étonner lorsque l'on connaît l'impact majeur que leurs travaux ont eu sur l'évolution de la réflexion à propos des sports et de la société contemporaine. Voir Norbert Elias et Eric Dunning, Sport et civilisation : la violence maîtrisée, traduit de l'anglais par Josette Chicheportiche et Fabienne Duvigneau, avant-propos de Roger Chartier, Paris, Fayard, 1994.
} 
historiographiques, études politiques, considérations sur l'évolution de l'éthique et de la morale associées au sport, récits biographiques, réflexions sur les idéologies et les imaginaires sociaux, comparaisons critiques entre les photos de sportifs publiées dans les journaux et les légendes qui les accompagnent, analyses de textes portant sur l'esthétique, la narratologie, la poétique, la stylistique, la sémiotique, la psychocritique et la sociocritique.

L'envers de cette profusion, certes stimulante pour l'intellectuel s'intéressant à la connexion mot-muscle, est qu'elle ne se double presque jamais d'une exploration en profondeur de chacune de ces avenues, qui sont trop rapidement évoquées. De même que Jean Durry, la plupart des auteurs se contentent, à partir du sujet dont ils traitent, de «brosser un rapide panorama et de proposer diverses pistes» (p. 10). Pierre Charreton précise ainsi, dès les premières phrases de son intervention, que ce dont il entend parler, à savoir les mutations idéologiques et esthétiques qui affectent les représentations du corps dans la littérature à thème sportif, déborde les limites d'un article: "Vaste sujet! Il y aurait tant à dire que je serai tenu de procéder souvent par allusion et avec une économie drastique de citations: chaque tête de paragraphe pourrait donner lieu à une conférence développée...» (p. 119) Plutôt que de livrer une synthèse courte et décevante de ses anciens travaux, qui ne leur apporte rien de neuf, comme il le fait ici, difficile de ne pas penser qu'il aurait mieux fait de choisir l'une des têtes de paragraphe dont il parle, par exemple «Le progrès physique de l'humanité » (p. 121), pour lui consacrer la «conférence développée» qu'elle méritait... Il y a bien, dans l'ensemble du volume, quelques communications qui abordent des sujets moins larges et qui débouchent sur des considérations originales. Ainsi, l'analyse que Birgit Wagner consacre à la signification idéologique et fantasmatique de la rencontre entre la machine, le corps et la compétition dans Le surmâle d'Alfred Jarry (p. 129-140) est éclairante, tout comme est captivant le récit, fait par Jean Touzot, des relations que Jean Cocteau entretint pendant plus d'un an avec le boxeur noir américain Alphonso «Panama Al» Brown (p. 153-161). En revanche, dire, comme le fait Pierre-Louis Rey, que Camus, joueur et grand amateur de foot, n'a pas beaucoup écrit sur ce sport parce 
qu'il se voulait un écrivain sérieux (p. 179-188), ou expliquer l'olympisme allégorique qui figure les camps de concentration dans $W$ on le souvenir d'enfance par le seul fait que Georges Perec aurait voulu mettre l'idéologie sportive à la question en rappelant que le régime hitlérien l'avait détournée à son profit (p. 201-207), voilà qui, dans un cas comme dans l'autre, réduit à bien peu de choses des cas de figure aussi passionnants que complexes.

Les différents raccourcis qui déparent le volume s'expliquent en partie par le choix éditorial de ne modifier en rien les dix-huit textes réunis ici, qui étaient au départ destinés à être communiqués lors d'un colloque. L'introduction s'intitule «Écrire le sport. Conférence en guise d'ouverture » (je souligne) et débute par une formule éminemment orale, "Chers amis » (p. 9). Dans son étude sur les mots du sport (p. 39-51), Jean-François Sablayrolles évoque les « autres orateurs» qui vont le suivre (p. 51, je souligne). Une formule telle que «En cette année hugolienne » (p. 10) est sans aucun doute à sa place dans une conférence donnée en 2002; elle l'est beaucoup moins dans un livre publié en 2005. Ce sont là de petits détails qui révèlent que ni les auteurs des différents articles ni les directeurs du collectif ne se sont souciés d'ajuster cette publication aux exigences de l'écrit qui, la plupart des universitaires le savent, ne sont pas les mêmes que celles de l'oral. Il aurait suffi, dans plusieurs des cas, d'ajouter quelques pages complétant ou nuançant les points que le temps souvent très bref imparti au participant d'un colloque ne lui permet pas de développer pour faire d'Écrire le sport un livre important sur le sujet encore trop peu étudié des relations qui se sont établies depuis au moins les cent cinquante dernières années entre écriture, représentations et pratiques sportives. En l'état actuel, ce livre est tout au plus - mais c'est déjà appréciable - une source d'informations bibliographiques considérable qui devrait donner envie, même aux plus érudits en la matière, de se lancer sans plus attendre dans un marathon de lectures.

Référence : Philippe Baudorre, Myriam Boucharenc et Michel Brousse (dir.), Écrire le sport, Pessac, Presses universitaires de Bordeaux, coll. «Regards croisés sur le sport », 2005, 246 p. 